\title{
Revisiting neutrino and sneutrino dark matter in natural SUSY scenarios
}

\author{
T. Faber, ${ }^{*}$ Y. Liu, ${ }^{\dagger}$ and W. Porod $\oplus^{\ddagger}$ \\ Institut für Theoretische Physik und Astrophysik, Uni Würzburg, \\ Emil-Hilb-Weg 22, D-97074 Würzburg, Germany \\ J. Jones-Pérez $\oplus^{\S}$ \\ Sección Física, Departamento de Ciencias, Pontificia Universidad Católica del Perú, \\ Apartado 1761, Lima, Peru
}

(Received 1 October 2019; accepted 3 March 2020; published 24 March 2020)

\begin{abstract}
We study natural supersymmetric scenarios with light right-handed neutrino superfields, and consider the possibility of having either a neutrino or a sneutrino as a dark matter candidate. For the former, we evaluate the possibility of having SUSY corrections on the $\nu_{4} \rightarrow \nu_{\ell} \gamma$ decay rate, such that the NuStar bounds are relaxed. We find that corrections are too small. For sneutrino dark matter, we consider thermal and nonthermal production, taking into account freeze-out, freeze-in, and super-weakly interacting massive particles mechanisms. For the nonthermal case, we find that the $\tilde{\nu}_{R}$ can reproduce the observed relic density by adjusting the R-sneutrino mass and Yukawa couplings. For the thermal case, we find the need to extend the model in order to enhance sneutrino annihilations, which we exemplify in a model with an extended gauge symmetry.
\end{abstract}

DOI: 10.1103/PhysRevD.101.055029

\section{INTRODUCTION}

In the past decades, supersymmetric (SUSY) models have been the most popular candidates for physics beyond the Standard Model (BSM). This popularity has been very well justified, given the capacity of the minimal supersymmetric Standard Model (MSSM) of solving the hierarchy problem, providing a dark matter (DM) candidate, and achieving gauge coupling unification, among other features.

Unfortunately, in the last years there has been a lack of statistically significant signals of new physics in both collider and dark matter experiments. These constraints affect all BSM models, and in the case of SUSY they imply that either the new particles are all much heavier than expected, or that the SUSY spectrum is much more complicated than what was initially expected. This situation motivates the relaxation of assumptions typically taken in past works, such as supergravity-inspired spectra, attempting at the same time to keep most of the attractive features of such models.

\footnotetext{
*thomas.faber@physik.uni-wuerzburg.de

†yang.liu@uni-wuerzburg.de

*porod@physik.uni-wuerzburg.de

jones.j@pucp.edu.pe
}

Published by the American Physical Society under the terms of the Creative Commons Attribution 4.0 International license. Further distribution of this work must maintain attribution to the author(s) and the published article's title, journal citation, and DOI. Funded by SCOAP.
In particular, we are interested in preserving naturalness as best as possible. This has important consequences, as we know from fine-tuning arguments [1] that the $\mu$ parameter should be close to the electroweak breaking scale. This implies that the lightest neutralinos $\tilde{\chi}_{1,2}^{0}$ and chargino $\tilde{\chi}_{1}^{ \pm}$ should be of Higgsino type, which leads to them being nearly mass degenerate [2].

Another feature that we wish to keep is having a good DM candidate. On the MSSM, the DM candidates are the lightest sneutrino $\tilde{\nu}_{L}$ and neutralino $\tilde{\chi}^{0}$, which are so-called weakly interacting massive particles (WIMPs). The $\tilde{\nu}_{L}$ has no difficulty in reproducing the correct relic density for $\mathrm{GeV}$-scale masses, but in this case the spin-independent DM-nucleon cross-section lies above the current bounds, such as XENON1T [3]. On the other hand, if the $\tilde{\chi}^{0}$ is of Higgsino type, one finds that for $\mathcal{O}(100 \mathrm{GeV})$-scale masses the correct relic density cannot be reproduced [4]. Given this situation, if we insist on having a natural SUSY solution to the DM problem, it is necessary to expand the model.

Motivated by this, we turn to one problem not addressed by the MSSM: the origin of neutrino masses. One of the most popular mechanisms addressing this issue is the type I seesaw [5-9], where new $\hat{\nu}_{R}$ superfields are added. In this case, we find ourselves with new candidates for DM: the new $\nu_{R}$ and $\tilde{\nu}_{R}$, provided the latter is the lightest supersymmetric particle $^{1}$ (LSP) [11-22].

\footnotetext{
${ }^{1}$ The scenario, where Higgsino dark matter can be obtained from late sneutrino decays has been investigated in Ref. [10].
} 
Having the $\tilde{\nu}_{R}$ as an LSP is also of phenomenological interest in the context of colliders [17,19,23-28]. In Ref. [26] it was found that there exists a region of the parameter space that has not been probed so far by the LHC, characterized by having a $\tilde{\nu}_{R}$ LSP, with light sleptons and Higgsinos, as well as heavy gauginos. This region was also characterized by having large $Y_{\nu}$ couplings, which are interesting for two reasons. First, having large Yukawa couplings allows us to avoid collider bounds on long-lived sleptons. And second, this case could be probed in future experiments, such as those searching for charged lepton flavor violation, lepton number violation, or heavy neutral leptons at colliders. It is thus of our interest to probe further this region of the parameter space, and find out what conditions do the $\nu_{R}$ and $\tilde{\nu}_{R}$ have to follow in order to reproduce the correct relic density. This is the main motivation behind this paper.

To this end, in Sec. II, we present the main features of a minimal model where only the $\hat{\nu}_{R}$ superfields are added. We study the conditions where thermal equilibrium can be attained, and explore thermal freeze-out as well as superWIMP and freeze-in mechanisms. In Sec. III we add a $U(1)_{B-L}$ gauge symmetry, which is spontaneously broken by additional superfields. We find the new superfields in this model can act as mediators which help the $\tilde{\nu}_{R}$ attain thermal equilibrium.

\section{MINIMAL SEESAW MODEL}

\section{A. Model definition}

As mentioned previously, we add three sterile neutrino superfields $\hat{\nu}_{R k}(k=4,5,6)$ to the MSSM particle content, and assume conserved $R$-parity. With this, the superpotential reads as

$\mathcal{W}_{\text {eff }}=\mathcal{W}_{\mathrm{MSSM}}+\frac{1}{2}\left(M_{R}\right)_{i j} \hat{\nu}_{R i} \hat{\nu}_{R j}+\left(Y_{\nu}\right)_{i j} \hat{L}_{i} \cdot \hat{H}_{u} \hat{\nu}_{R j}$.

The corresponding soft SUSY breaking terms are given by

$$
\begin{aligned}
\mathcal{V}^{\text {soft }}= & \mathcal{V}_{\text {MSSM }}^{\text {soft }}+\left(m_{\tilde{\nu}_{R}}^{2}\right)_{i j} \tilde{\nu}_{R i}^{*} \tilde{\nu}_{R j} \\
& +\left(\frac{1}{2}\left(B_{\tilde{\nu}}\right)_{i j} \tilde{\nu}_{R i} \tilde{\nu}_{R j}+\left(T_{\nu}\right)_{i j} \tilde{L}_{i} \cdot H_{u} \tilde{\nu}_{R j}+\text { H.c. }\right) .
\end{aligned}
$$

For only one family of $\hat{\nu}_{L} / \hat{\nu}_{R}$, the seesaw mechanism determines the size of the Yukawa couplings in terms of the neutrino masses, $Y_{\nu} \sim(\sqrt{2 m M}) / v_{u}$. If the heavy neutrino masses $M$ are on the $\mathrm{GeV}$ scale, one expects very small Yukawa couplings. For example, assuming a light neutrino mass $m_{1}=10^{-3} \mathrm{eV}$ and $\tan \beta=6$, then, for $M=1 \mathrm{GeV}$ we have $Y_{\nu}=6 \times 10^{-9}$.

Nevertheless, when having more than one generation of $\hat{\nu}_{R}$, it is possible to enhance the Yukawas. In this case, it is useful to implement a Casa-Ibarra-like parametrization $[29,30]$. This describes neutrino mixing in terms of the active-light mixing matrix $U_{\text {PMNS }}$, all six neutrino masses, and the orthogonal $R$ matrix:

$R=\left(\begin{array}{ccc}c_{45} & s_{45} & 0 \\ -s_{45} & c_{45} & 0 \\ 0 & 0 & 1\end{array}\right)\left(\begin{array}{ccc}c_{46} & 0 & s_{46} \\ 0 & 1 & 0 \\ -s_{46} & 0 & c_{46}\end{array}\right)\left(\begin{array}{ccc}1 & 0 & 0 \\ 0 & c_{56} & s_{56} \\ 0 & -s_{56} & c_{56}\end{array}\right)$,

where $s_{i j}$ and $c_{i j}$ are the sines and cosines of new complex angles, $\rho_{i j}+i \gamma_{i j}$. The $\gamma_{i j}$ lead to hyperbolic functions, which exponentially enhance the Yukawas.

If only one of the $\gamma_{i j}$ is nonzero, the Yukawas can be expressed in a simple way. For example, by taking only $\gamma_{56}$ different from zero, and normal mass ordering, we find:

$$
\begin{gathered}
\left(Y_{\nu}\right)_{a 4}=\left(U_{\mathrm{PMNS}}\right)_{a 1} \sqrt{\frac{2 m_{1} M_{4}}{v_{u}^{2}}} \\
\left(Y_{\nu}\right)_{a 5}=-i \operatorname{sgn}\left(\gamma_{56}\right)\left(Z_{a}^{\mathrm{NO}}\right) \sqrt{\frac{2 m_{3} M_{5}}{v_{u}^{2}}} \cosh \gamma_{56} \\
\left(Y_{\nu}\right)_{a 6}=\left(Z_{a}^{\mathrm{NO}}\right) \sqrt{\frac{2 m_{3} M_{6}}{v_{u}^{2}}} \cosh \gamma_{56}
\end{gathered}
$$

$\left(Z_{a}^{\mathrm{NO}}\right)=\left(U_{\mathrm{PMNS}}\right)_{a 3}+i \operatorname{sgn}\left(\gamma_{56}\right) \sqrt{\frac{m_{2}}{m_{3}}}\left(U_{\mathrm{PMNS}}\right)_{a 1}$

where $a=e, \mu, \tau$, and $m_{1}, m_{2}, m_{3}\left(M_{4}, M_{5}, M_{6}\right)$ are the light (heavy) neutrino masses. Notice that only the couplings of $\hat{\nu}_{R 5}$ and $\hat{\nu}_{R 6}$ are enhanced, with $\hat{\nu}_{R 4}$ following the standard seesaw expectation. By taking $\gamma_{56} \approx 3,5.3,7.6$, 9.9, the elements $Y_{a 5}$ and $Y_{a 6}$ are enhanced by a factor $10,10^{2}, 10^{3}$ and $10^{4}$, respectively. Switching on the other angles does not change the generic feature that the couplings of two of the heavy neutrinos are enhanced with respect to the third one. This is a consequence of the fact that one needs an even number of right-handed neutrinos forming pseudo-Dirac neutrinos. In the following, we shall use this scenario, setting all other $\gamma_{i j}$ and $\rho_{i j}$ to zero.

It is well known that when enhancing the heavy neutrino couplings one can have large contributions to neutrinoless double beta decays from $\nu_{R 5}$ and $\nu_{R 6}$. The nonobservation of this process forces the latter to have almost degenerate masses, $M_{5} \approx M_{6}$ [31-34], that is, they form a pseudoDirac pair. This statement holds in the presence of $R$-parity conserving SUSY, as no tree-level SUSY contributions to this process exist.

Let us now turn to the $\tilde{\nu}$ sector. As was discussed in [26], if we neglect $B_{\tilde{\nu}}$ and $Y_{\nu} M_{R}^{\dagger}$ terms, and assume vanishing $C P$-violation in the sneutrino mass matrix, the real and imaginary parts of the sneutrino fields remain aligned. This means that we can work directly with complex $\left(\tilde{\nu}_{L}, \tilde{\nu}_{R}\right)$, with the mass matrix having the following leading terms: 
TABLE I. Fixed parameters in neutrino (top), and SUSY (bottom) sectors.

\begin{tabular}{lccc}
\hline \hline Parameter & Value & Parameter & Value \\
\hline$s_{12}^{2}$ & 0.310 & $m_{1}$ & $10^{-3} \mathrm{eV}$ \\
$s_{13}^{2}$ & 0.0224 & $\Delta m_{21}^{2}$ & $7.39 \times 10^{-5} \mathrm{eV}^{2}$ \\
$s_{23}^{2}$ & 0.582 & $\Delta m_{31}^{2}$ & $2.525 \times 10^{-3} \mathrm{eV}^{2}$ \\
& & $M_{5}=M_{6}$ & $5 \mathrm{GeV}$ \\
$\mu$ & $400 \mathrm{GeV}$ & $M_{1}=M_{2}=M_{3}$ & $\mathcal{O}(10 \mathrm{TeV})$ \\
$T_{\nu}$ & $0 \mathrm{GeV}$ & $B_{\nu}$ & $0 \mathrm{GeV}^{2}$ \\
& & $\tan \beta$ & 6 \\
\hline \hline
\end{tabular}

$M_{\tilde{\nu}}^{2}=\left(\begin{array}{cc}m_{\tilde{L}}^{2}+\frac{1}{2} m_{Z}^{2} \cos 2 \beta & \frac{v_{u}}{\sqrt{2}}\left(T_{\nu}-\mu Y_{\nu} \cot \beta\right) \\ \frac{v_{u}}{\sqrt{2}}\left(T_{\nu}^{T}-\mu Y_{\nu}^{T} \cot \beta\right) & m_{\tilde{\nu}_{R}}^{2}+M_{R}^{T} M_{R}\end{array}\right)$.

Diagonalization of this mass matrix leads to the lightest mass eigenstate $\tilde{\nu}_{1}$, which in our framework is the LSP. The $\tilde{\nu}_{1}$ state will be a superposition of $\tilde{\nu}_{R}$ and $\tilde{\nu}_{L}$. Thus, for one generation, we can have $\tilde{\nu}_{1}=\cos \tilde{\theta} \tilde{\nu}_{R}+\sin \tilde{\theta} \tilde{\nu}_{L}$ with L-R mixing angle:

$$
\tan 2 \tilde{\theta} \sim \frac{v_{u} Y_{\nu}}{\sqrt{2}} \frac{A_{\nu}-\mu \cot \beta}{m_{\tilde{\nu}_{L}}^{2}-m_{\tilde{\nu}_{R}}^{2}+\frac{1}{2} m_{Z}^{2} \cos 2 \beta-M_{R}^{2}} .
$$

Here we have defined $T_{\nu} \equiv Y_{\nu} A_{\nu}$. The mixing is strongly suppressed by $Y_{\nu}$ and, given our assumptions on the size of $\mu$, can only be enhanced by taking a very large $A_{\nu}$, or tuning the masses such that the denominator vanishes. In the following we shall not consider any of these possibilities, such that all L-R mixing effectively vanishes.

In this work, for definiteness, we take the neutrino and SUSY parameters as shown in Table I, with all $C P$ phases equal to zero. Oscillation parameters can be found in [35,36]. $M_{4}$ will be allowed to vary between $1 \mathrm{keV}$ and $1 \mathrm{GeV}$ within our results. For the other heavy neutrino masses given in Table I, direct search bounds [37] restrict $\gamma_{56} \lesssim 8$. Taking as example $\gamma_{56}=3(7)$, we find $\left|\left(Y_{\nu}\right)_{a 5}\right|=$ $\left|\left(Y_{\nu}\right)_{a 6}\right| \sim 10^{-7}\left(10^{-5}\right)$. On the sneutrino soft sector, only $m_{\tilde{L}}$ and $m_{\tilde{\nu}_{R}}$ are nonzero. Given the strong flavor constraints coming from processes such as $\mu \rightarrow e \gamma$, we take the soft masses flavor diagonal. This implies that all mixing effects are negligible, allowing us to identify the mass eigenstates with the interaction eigenstates. In particular, $\tilde{\nu}_{1}$ will be one of the three $\tilde{\nu}_{R}$ states.

\section{B. Neutrino dark matter}

It is well known that a sterile neutrino with mass in the $\mathrm{keV}$ range can serve as potential DM candidate via oscillations with the left-handed neutrinos [38,39]. However, it is unstable, as it can decay into the lighter active neutrinos through the following processes:

$$
\nu_{4} \rightarrow \nu_{i} \gamma
$$

TABLE II. Predicted $\sin ^{2} 2 \theta$, for different values of $M_{4}$.

\begin{tabular}{lc}
\hline \hline$M_{4}(\mathrm{keV})$ & $\sin ^{2} 2 \theta$ \\
\hline 7 & $1.4 \times 10^{-7}$ \\
30 & $3.3 \times 10^{-8}$ \\
50 & $2.0 \times 10^{-8}$ \\
\hline \hline & \\
& $\rightarrow \nu_{i} \nu_{j} \nu_{k} \quad(i, j, k=1, \ldots, 3)$
\end{tabular}

The first final state can be detected via the resulting photon, e.g., by cosmological observations. In particular, data from the NuStar collaboration puts severe constraints on the allowed parameter space, as has been shown recently in [40]. From their Fig. 5, considering only the usual $W$-l-loop mediated contribution, one gets an upper bound for the quantity

$$
\sin ^{2} 2 \theta=\sum_{a=1}^{3}\left|U_{a 4}\right|^{2}
$$

of few times $10^{-11}$ for $M_{4}=7 \mathrm{keV}$ and up to around $10^{-14}$ for $M_{4}=50 \mathrm{keV}$. We find that the $\sin ^{2} 2 \theta$ predicted in our scenario is always well above the corresponding limit, as can be seen from the values given in Table II (see also [41] for a recent discussion on the seesaw prediction).

Still, one might wonder if the branching ratio into the photon could be modified via loops containing SUSY particles, ${ }^{2}$ such as those shown in Fig. 1. We have varied the soft SUSY parameters in the ranges $450 \leq m_{\tilde{L}} \leq 1000 \mathrm{GeV}$, $200 \leq m_{\tilde{E}} \leq 1000 \mathrm{GeV}, \quad-1 \leq A_{\tau} \leq 1 \mathrm{TeV}, \quad 200 \leq M_{2} \leq$ $1000 \mathrm{GeV}, 5 \leq \tan \beta \leq 40$, as well as $100 \leq|\mu| \leq 500 \mathrm{GeV}$. We find at most a 10 percent variation in the branching ratio for scenarios with light sleptons and charginos, and small $\tan \beta$. The main reason for this is due to the experimental bounds on the masses of the SUSY particles, which imply that the corresponding loops are suppressed with respect to the $W-l$ contributions. Thus, we conclude that the presence of SUSY does not affect the NuStar bounds.

In light of this negative result, one has two options to avoid the bounds. The first option is to increase $M_{4}$ above $700 \mathrm{MeV}$, such that $\nu_{4}$ decays quickly, without affecting big bang nucleosynthesis (BBN) [43]. The second option is to decrease $m_{1}$ under $10^{-9} \mathrm{eV}$, which suppresses the $\nu_{4}$ Yukawa coupling [see Eq. (2.4)]. This in turn decreases the mixing with the active neutrinos. Nevertheless, in both options $\nu_{4}$ can no longer be a good dark matter candidate, in the first case it decays too soon, while on the second case it is unlikely to be produced ${ }^{3}$ via oscillations with the

\footnotetext{
${ }^{2}$ For the calculation we have used the formulas given in [42] for the $H^{ \pm}-\tilde{\chi}_{i}^{\mp}$ loops for $\tilde{\chi}_{i}^{0} \rightarrow \tilde{\chi}_{j}^{0} \gamma$ substituting correspondingly masses and couplings.

${ }^{3}$ One might wonder if the presence of SUSY could lead to more efficient production processes, for example due to $\tilde{\nu}_{R}$ decays [44]. We have checked in [26] that in our model SUSY particles have negligible branching ratios into $\nu_{R}$ states, so one can safely disregard heavy neutrino production through SUSY decays.
} 

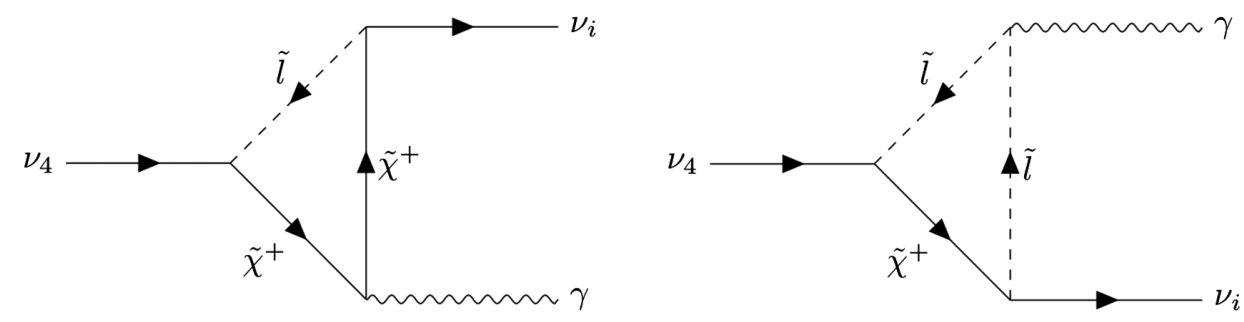

FIG. 1. Contributions to $\nu_{4} \rightarrow \nu_{i} \gamma$ from $\tilde{\chi}^{+} \tilde{l}$ loops.

left-handed neutrinos $[38,39]$. We thus discard $\nu_{4}$ as a candidate for dark matter in this model.

\section{Sneutrino dark matter}

The calculation of the $\tilde{\nu}_{1}$ relic density depends on whether it is in thermal equilibrium with the primordial plasma, or not. For this to happen, one requires that at some temperature $T$ we have:

$$
H(T)<\langle\sigma v\rangle_{T} n(T),
$$

where $H(T)$ is the Hubble constant, $\langle\sigma v\rangle_{T}$ is the thermal average of the cross section times velocity, and $n(T)$ is the number density of the $\tilde{\nu}_{1}$. Ultimately, a relevant factor in this condition is the size its couplings with other thermal particles, which are in turn determined by the size of the $Y_{\nu}$.

If the $\tilde{\nu}_{1}$ couplings are large enough such that it can be in thermal equilibrium at some temperature, the final value of $\Omega h^{2}$ follows the freeze-out mechanism, see, e.g., [45] and refs. therein. Here, the expansion of the Universe leads to a point where Eq. (2.13) does not hold, and the $\tilde{\nu}_{1}$ decouples from the plasma. The relic density depends on the number density at the freeze-out temperature, as well as on (co) annihilations with other thermal particles.

If the couplings are too small, the $\tilde{\nu}_{1}$ relic density proceeds from decays or annihilation of other particles in the primordial plasma. In the former case, the value of the relic density is mainly due to decays of the next to lightest SUSY particle (NLSP). For example, thermal neutralinos could decay after they freeze-out via $\tilde{\chi}^{0} \rightarrow \tilde{\nu}_{1} \nu$. In this situation the $\tilde{\nu}_{1}$ is said to follow the super-WIMP mechanism [12,46-48], and its relic density is proportional to the NLSP yield after freeze-out. In contrast, if the annihilations are more important than decays, the mechanism is that of freeze-in [49]. Here, the $\tilde{\nu}_{1}$ is created throughout the thermal history of the Universe by annihilations of all thermal particles, not only the NLSP. In both of the scenarios described above, the $\tilde{\nu}_{1}$ is called a feebly interacting massive particle (FIMP), and one assumes that it was not generated by other means at earlier times, such as during an inflationary period.

The naive seesaw expectation is that a light $\tilde{\nu}_{1}$ should have very small $Y_{\nu}$ couplings, and should thus be nonthermal. An important result of this procedure is that the NLSP can be long lived, which can lead to displaced vertices, disappearing tracks or heavy metastable charged particle signals at colliders $[19,23,50]$.

If the neutrino mass generation mechanism allows for larger Yukawas, the $\tilde{\nu}_{R}$ can be thermal [13]. From Eq. (2.13), we see that as long as $\langle\sigma v\rangle_{T} n(T) / H(T)>1$ for some value of $T$, these particles will have been in thermal equilibrium at some point in the history of the Universe. Thus, as a first step, we evaluate the required size of $Y_{\nu}$ for this condition to hold.

To this end, we used SARAH4.14.0 [51-55] to implement the model of [26] in SPheno 4.0.3 [56,57], which calculates the mass spectrum and branching ratios. We used SSP1.2.5 [58] to carry out the parameter variation. With the output, the variable $\langle\sigma v\rangle_{T} n(T) / H(T)$ was calculated with micrOMEGAs 5.0.9 [59]. Here one has to keep two details in mind. First of all, the program only provides $\langle\sigma v\rangle_{T}^{\text {ann }}$, the (co-)annihilation of $\tilde{\nu}_{R}$ with themselves and other SUSY particles into SM final states. In addition, micrOMEGAs provides the total $n_{\text {eq }}(T)$ from all SUSY particles, instead of the exclusive one for $\tilde{\nu}_{1}$. We do not consider this a serious problem, as $n_{\text {eq }}(T)$ is dominated by the LSP. Still, in order to be certain of our results, in the following we shall consider $\langle\sigma v\rangle_{T}^{\text {ann }} n_{\mathrm{eq}}(T) / H(T)<0.1$ as nonthermal, and $\langle\sigma v\rangle_{T}^{\mathrm{ann}} n_{\mathrm{eq}}(T) / H(T)>10$ as thermal.

Our results are shown in Fig. 2, where we evaluate $\langle\sigma v\rangle_{T}^{\mathrm{ann}} n_{\mathrm{eq}}(T) / H(T)$ as a function of $T$. Curves are shown for different SUSY masses, as well as different Yukawa couplings. We compare $Y_{\nu}^{\max } \sim \mathcal{O}\left(10^{-7}\right)\left(\gamma_{56}=3\right)$ with $Y_{\nu}^{\max } \sim \mathcal{O}\left(10^{-5}\right)\left(\gamma_{56}=7\right)$ on the left and right panels, respectively. We fix the SUSY masses as in Tab. I, and probe $\tilde{\nu}_{R}$ soft masses of 100, 200 and $300 \mathrm{GeV}$ (blue, green and orange, respectively). We also include MSSM slepton soft masses between 150 and $450 \mathrm{GeV}$, with the restriction $m_{\tilde{L}}=m_{\tilde{E}}>m_{\tilde{\nu}_{R}}$, such that the R-sneutrino is always the LSP.

In the figure, we find that all curves of $\langle\sigma v\rangle_{T}^{\mathrm{ann}} n_{\text {eq }}(T) / H(T)$ increase very quickly with $T$ up to a peak, with a subsequent drop as temperatures increase. This decrease is not surprising, since $H \sim T^{2}, n_{\mathrm{eq}} \sim T^{3}$ and $\langle\sigma v\rangle_{T}$ is expected to fall as $1 / T^{2}$ for large temperatures. The conclusions of the figure are straightforward. We find that, as long as $Y_{\nu}^{\max } \gtrsim \mathcal{O}\left(10^{-5}\right)$, a $\tilde{\nu}_{R}$ can be thermal. This conclusion is valid for $\mathcal{O}(100 \mathrm{GeV})$ scale R-sneutrino and L-slepton masses. For lower values of $Y_{\nu}$ a more careful 

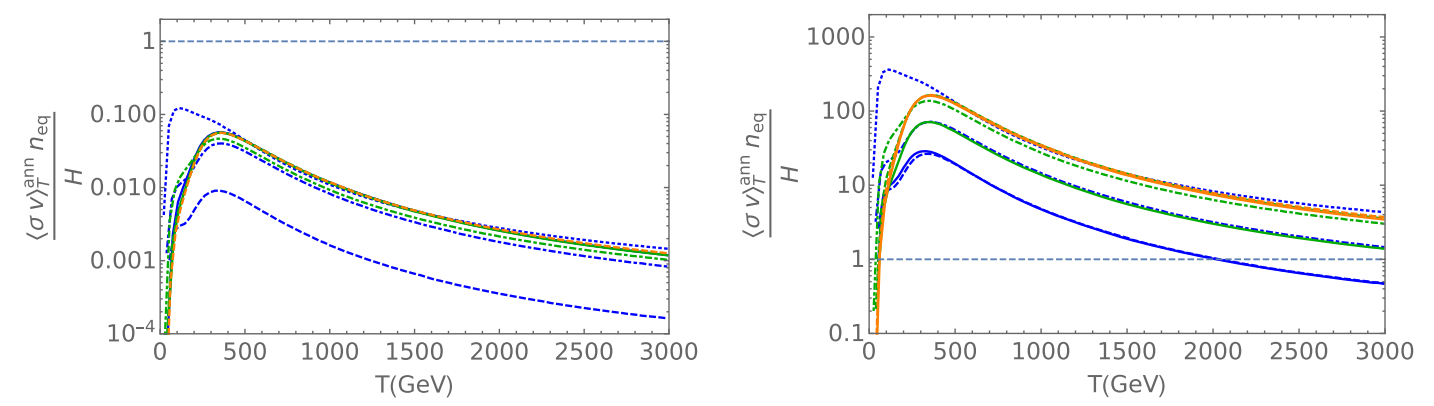

FIG. 2. Values of $\langle\sigma v\rangle_{T}^{\mathrm{ann}} n_{\mathrm{eq}}(T) / H(T)$ as a function of the temperature, for $Y_{\nu}^{\mathrm{max}} \sim \mathcal{O}\left(10^{-7}\right)\left(\mathcal{O}\left(10^{-5}\right)\right)$ on the left (right). Blue, green, and orange lines have $m_{\nu_{R}}=100,200,300 \mathrm{GeV}$, respectively. L-slepton masses of 150, 250, 350, $450 \mathrm{GeV}$ are shown in dotted, dotdashed, dashed, and solid lines, if applicable.

analysis would be necessary, unless $Y_{\nu}^{\max } \lesssim \mathcal{O}\left(10^{-7}\right)$, where we find no temperature which satisfies the condition $\langle\sigma v\rangle_{T}^{\mathrm{ann}} n_{\mathrm{eq}}(T) / H(T)>1$ and the $\tilde{\nu}_{R}$ is certainly a FIMP.

In the following, we explore the status of DM in the setup of Ref. [26]. As mentioned in the Introduction, we seek to enhance Yukawa couplings in order to avoid bounds on long-lived particles, and to hope for experimental correlations between the $\nu_{R}$ and $\tilde{\nu}_{R}$ sectors. To achieve this, we set $\gamma_{56}=7$, so $\tilde{\nu}_{R 5}$ and $\tilde{\nu}_{R 6}$ are thermal particles. For simplicity, we also fix $m_{\tilde{\nu}_{R 5}}=m_{\tilde{\nu}_{R 6}}$. In contrast, the remaining eigenstate $\tilde{\nu}_{R 4}$ is left with small Yukawa couplings, such that it is a nonthermal $\tilde{\nu}_{R}$.

If the $\tilde{\nu}_{R 4}$ mass is larger than that of $\tilde{\nu}_{R 5}$ or $\tilde{\nu}_{R 6}$, then the latter is a thermal LSP, and the relic density $\left(\Omega h^{2}\right)^{\text {th }}$ is generated via freeze-out. In this case we find that the value of $\left(\Omega h^{2}\right)^{\text {th }}$ calculated by micrOMEGAs is extremely large, ruling out the whole parameter space.

Alternatively, if $\tilde{\nu}_{R 4}$ is the LSP, the relic density is obtained from a combination of super-WIMP $\left(\Omega h^{2}\right)^{\operatorname{dec}}$ and freeze-in $\left(\Omega h^{2}\right)^{\text {in }}$ processes. ${ }^{4}$ The super-WIMP contribution is obtained from ${ }^{5}$ :

$$
\left(\Omega h^{2}\right)^{\mathrm{dec}}=\left(\Omega h^{2}\right)^{\mathrm{th}} \frac{m_{\tilde{\nu}_{1}}}{m_{\mathrm{NLSP}}^{\text {th }}}
$$

where $m_{\mathrm{NLSP}}^{\text {th }}$ is the mass of the lightest thermal SUSY partner. Thus, one can adjust this contribution to any desired value by choosing an appropriate $m_{\tilde{\nu}_{1}}$. In this case it is not necessary for the thermal $\tilde{\nu}_{R}$ to be NLSP, it is perfectly possible to have a thermal $\tilde{\nu}_{L}$ or $\tilde{h}$ NLSP which later decays to the $\tilde{\nu}_{1}$.

The super-WIMP contribution by itself cannot explain the observed relic density in two situations. First, since $\left(\Omega h^{2}\right)^{\text {dec }} \leq\left(\Omega h^{2}\right)^{\text {th }}$, it is impossible to get the correct relic density if $\left(\Omega h^{2}\right)^{\text {th }}$ is too small (this can be the case for both $\tilde{\nu}_{L}$ and $\tilde{h}$ NLSP). Second, it is also possible for $\left(\Omega h^{2}\right)^{\text {th }}$ to

\footnotetext{
${ }_{5}^{4}$ For the case of a generic fermionic DM candidate see [60].

5 micrOMEGAs assumes that the decay products of new non-DM particles thermalize instantly, so entropy-dilution effects such as those in [61] are not included.
}

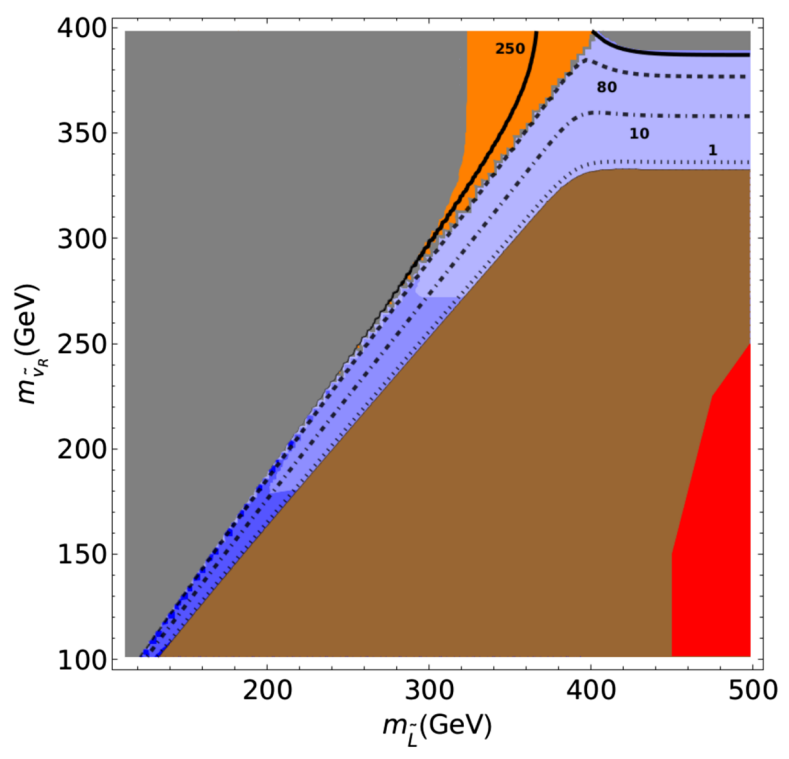

FIG. 3. Maximum possible mass of feebly interacting sneutrino, such that $\left(\Omega h^{2}\right)^{\text {dec }}$ matches the observed relic density. Masses of 250, 80, 10 and $1 \mathrm{GeV}$ are shown in solid, dashed, dashed-dotted, and dotted black lines. Lifetimes smaller than $10^{4}$, $10^{5}$, and $10^{6}$ seconds are shown in light blue, blue, and dark blue, respectively. Brown and red regions are excluded, gray regions cannot reproduce the correct relic density using super-WIMP mechanism. More details are given in the text.

be so large that the feeble $\tilde{\nu}_{1}$ mass must be lower than the $\nu_{4}$ mass. This situation would require a negative soft $m_{\nu_{R}}^{2}$, which we shall discard. ${ }^{6}$ Nevertheless, only the latter case is a problem, since a small $\left(\Omega h^{2}\right)^{\text {dec }}$ can be complemented by a larger $\left(\Omega h^{2}\right)^{\text {in }}$.

We show the results of this super-WIMP-only analysis in Fig. 3. We vary $m_{\tilde{L}}=m_{\tilde{E}}$ and $m_{\tilde{\nu}_{R}} \equiv m_{\tilde{\nu}_{R 5,6}}$, which are the

\footnotetext{
${ }^{6}$ Strictly speaking, this only true in case of diagonal $m_{\tilde{\nu}_{R}}^{2}$. However, to decrease the $\tilde{\nu}_{R 4}$ mass below $M_{4}$ would require very large off-diagonals, with the risk of giving large couplings to all $\tilde{\nu}_{R}$ and/or substantial contributions to lepton flavor violating observables, which are severely bound by experimental data.
} 
soft masses of thermal particles, and assume $m_{1}=10^{-3} \mathrm{eV}$ and $M_{4}=700 \mathrm{MeV}$. The black contour lines shown the maximum allowed mass of $\tilde{\nu}_{1}$, such that the superWIMP mechanism saturates the relic density, that is, $\left(\Omega h^{2}\right)^{\text {dec }}=0.12$. We find that $m_{\tilde{\nu}_{1}}$ spans from $700 \mathrm{MeV}$ to almost $300 \mathrm{GeV}$.

On the gray regions of the figure, the thermal L-sleptons and Higgsinos have large (co)annihilation cross sections, leading to a too small $\left(\Omega h^{2}\right)^{\text {th }}$. This means that the superWIMP mechanism cannot explain by itself the observed relic density, so there is no upper bound on $m_{\tilde{\nu}_{1}}$ apart from the requirement of being the LSP.

The brown and red regions are excluded. On the brown region, the feeble $\tilde{\nu}_{1}$ requires a mass lower than the minimum of $M_{4}=700 \mathrm{MeV}$, so $\left(\Omega h^{2}\right)^{\mathrm{dec}}$ is too big. Moreover, the red region was ruled out by LHC searches in Ref. [26]. With this, we conclude that if the super-WIMP mechanism was entirely responsible for dark matter production, then it would only be viable in a reduced region of the evaluated parameter space.

The main annihilation channels giving $\left(\Omega h^{2}\right)^{\text {th }}$ vary throughout the figure. For $m_{\tilde{L}}$ below $\mu=400 \mathrm{GeV}$, $\tilde{\nu} \tilde{\nu}$ annihilation dominates, mainly into $W^{+} W^{-}, Z Z$, and $t \bar{t}$ final states. Above $400 \mathrm{GeV}$, neutralino and chargino (co) annihilation determine the thermal relic density, with channels into $u_{i} d_{i}$ and $e_{i} \nu_{j}$ final states giving the largest contributions.

Due to $\tilde{\nu}_{1}$ being a FIMP, it is important to be aware of the thermal NLSP lifetime. If longer than one second, it can be subject to BBN constraints [19,62-64]. This information is included in Fig. 3. On the figure, the blue regions feature $\tilde{\nu}_{R 5,6}$ NLSP lifetimes between $10^{3}$ and $10^{6}$ seconds. On the orange region, the $\tilde{\nu}_{L}$ is the NLSP, with a lifetime smaller than one second. In the gray regions we find that, for a $700 \mathrm{MeV} \tilde{\nu}_{1}$, the lifetime of the $\tilde{\nu}_{L}$ NLSP (left region) is also lower than a second. However, on the upper right gray region we again have a $\tilde{\nu}_{R}$ NLSP, with a lifetime of around $10^{2} \mathrm{~s}$.

It is important to take into account that this information on the lifetime is strictly valid for the selected values of $m_{1}$ and $M_{4}$, since the $\left|\left(Y_{\nu}\right)_{a 4}\right|^{2}$ coupling of $\tilde{\nu}_{R 4}$ depend on them [see Eq. (2.4)]. For smaller $m_{1}$ or $M_{4}$, it will increase. We briefly discuss the impact of the NLSP lifetime on BBN further below.

We now turn to the calculation of the freeze-in contribution, $\left(\Omega h^{2}\right)^{\mathrm{fi}}$, which is again calculated with micrOMEGAs. Throughout the evaluated parameter space, we find that if we take $m_{\tilde{\nu}_{1}}$ equal to the maximum value allowed by the superWIMP mechanism, the freeze-in contribution can greatly exceed the observed value.

One way of suppressing freeze-in is by reducing the lightest neutrino mass $m_{1}$, which decreases $\left(Y_{\nu}\right)_{a 4}$. Due to the long calculation time, we show results for only one representative point in Fig. 4, with $M_{4}=700 \mathrm{MeV}, m_{\tilde{L}}=$ $323 \mathrm{GeV}$ and $m_{\tilde{\nu}_{R}}=302 \mathrm{GeV}$, where $\tilde{\nu}_{R 5}$ is the NLSP. We

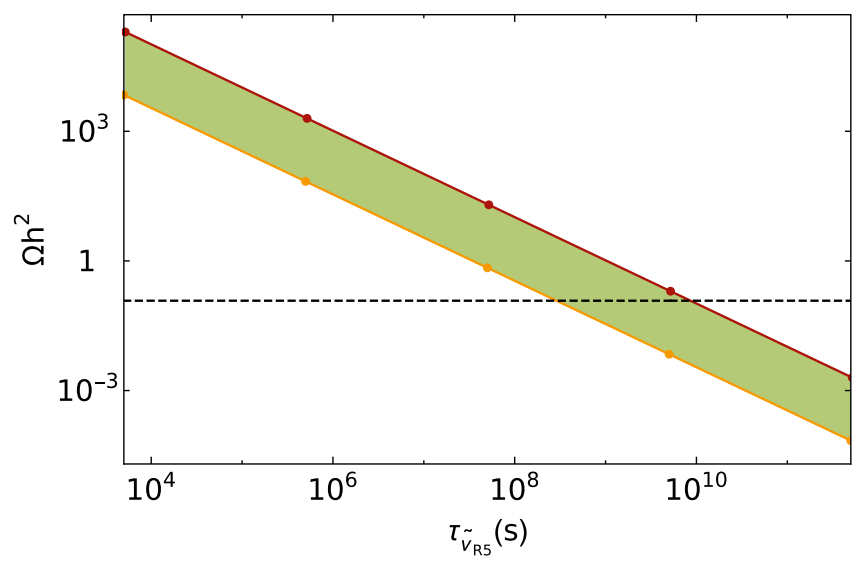

FIG. 4. Freeze-in contribution to our test point. Red (yellow) curve shows contributions for maximum (minimum) $\tilde{\nu}_{1}$ mass. Dashed line gives observed $\Omega h^{2}$. The dots indicate $m_{1}=10^{-3}, 10^{-5}, 10^{-7}, 10^{-9}$, and $10^{-11} \mathrm{eV}$ from left to right.

see that reducing $\left(Y_{\nu}\right)_{a 4}$ increases the $\tilde{\nu}_{R 5}$ lifetime, as shown in the red curve of the figure. Given the large lifetime, assuming that BBN constraints are avoided, it is still important to check that the time for recombination $\sim 10^{13} \mathrm{~s}$ is not exceeded, else the $\tilde{\nu}_{R 5}$ would decay into $\tilde{\nu}_{1}$ after the cosmic microwave background was emitted. In this case, the relic density observed by Planck [65] would correspond to $\left(\Omega h^{2}\right)^{\text {th }}$, ruling out the scenario. For our example, we find that $\left(\Omega h^{2}\right)^{\mathrm{fi}}$ becomes subdominant when $m_{1} \lesssim 10^{-9} \mathrm{eV}$, and that the recombination constraint is satisfied. This solution is also attractive as no additional $\nu_{R}$ DM is produced, since both $M_{4}$ is large and $\left(Y_{\nu}\right)_{a 4}$ is tiny.

Alternatively, it is possible to have a smaller $m_{\tilde{\nu}_{1}}$, such that the super-WIMP contribution is negligible. In this case, one needs to adjust the lightest neutrino mass, such that $\left(\Omega h^{2}\right)^{\mathrm{fi}}$ reproduces the observations. Such a scenario is shown on the yellow curve of Fig. 4, where we have chosen $m_{\tilde{\nu}_{1}}=M_{4}=700 \mathrm{MeV}$. Here one obtains the correct relic density when $m_{1} \approx \mathcal{O}\left(10^{-8}\right) \mathrm{eV}$.

Thus, we find that in this scenario, where $\left(Y_{\nu}\right)_{a 4}$ is suppressed and $\left(Y_{\nu}\right)_{a 5},\left(Y_{\nu}\right)_{a 6}$ are enhanced, the lightest $\mathrm{R}$-sneutrino can reproduce the observed relic density, with a dominant super-WIMP or freeze-in mechanism depending on $m_{\tilde{\nu}_{1}}$ and $\left(Y_{\nu}\right)_{a 4}$. However, regardless of the dominating mechanism, the NLSP lifetime is considerably large, and needs to pass BBN constraints. As the $\tilde{\nu}_{R 5}$ has no hadronic decays, this model does not lead to any any hadro-dissociation nor $p \leftrightarrow n$ conversion processes. However, around $50 \%$ of the decays are into charged leptons, so it could be subject to photo-dissociation processes. According to [64], these are important for lifetimes above $10^{4}-10^{6}$ seconds. Even though a solid conclusion can only be obtained through a detailed numerical simulation, which is outside the scope of this work, we can conclude that BBN constraints suggest that the $\tilde{\nu}_{R 5}$ density, and thus 
$\left(\Omega h^{2}\right)^{\text {th }}$, would need to be heavily suppressed. If confirmed, the only way to have R-sneutrino dark matter would be to either have a $\tilde{\nu}_{L}$ NLSP with a short lifetime (orange and upper left gray regions of Fig. 3) or to restrict ourselves to the upper right gray region of the figure where $\left(\Omega h^{2}\right)^{\text {th }}$ is small. Alternatively, we could decrease $\left(Y_{\nu}\right)_{a 5}$ and $\left(Y_{\nu}\right)_{a 6}$ such that no $\tilde{\nu}_{R}$ is thermal.

As a final remark, we must comment that other works attempted to have a thermal $\tilde{\nu}_{R}$ by generating a large mixing with the $\tilde{\nu}_{L}[14,16,17,27]$. The main idea was to allow the mass eigenstate to interact via gauge interactions, with a relative suppression of the overall coupling due to the mixing. This would allow the sneutrino to remain thermal, but at the same time avoid direct detection bounds. For this approach to work, it was required to have $\sin \tilde{\theta} \gtrsim 10^{-2}$.

As we see from Eq. (2.9), the L-R mixing is suppressed by $Y_{\nu}$. It is clear that even if we increased $\gamma_{i j}$ to their maximum values allowed by experiment, an additional enhancement is required to bring the mixing to the required level. Earlier works have considered an extremely large $A_{\nu}$, such that $Y_{\nu} A_{\nu}$ becomes of the order of the other soft terms [66], e.g., the slepton mass parameters. However, this creates the danger of a charge breaking minimum similar to the well-known problem of charge and color breaking minima within the MSSM, see, e.g., [67] for an extensive discussion on tree-level constraints.

In our case, we need to assess if the addition of the new $\hat{\nu}_{R}$ can lead scalar fields other than the Higgs to acquire a vacuum expectation value (vev). In particular, we will evaluate the following vev pattern for one generation of sleptons and sneutrinos:

$$
\left\langle H^{+}\right\rangle=\left\langle\tilde{l}_{L}^{-}\right\rangle=\left\langle\tilde{\nu}_{R}\right\rangle=\alpha,
$$

as this corresponds to a $D$-flat direction in the scalar potential. Assuming that all other fields do not acquire a vev and that all parameters are real, one gets for the potential at tree level

$$
\begin{aligned}
V= & \left(m_{H_{u}}^{2}+\mu^{2}+m_{\tilde{L}}^{2}+m_{\tilde{\nu}_{R}}^{2}+M_{R}^{2}+B_{\tilde{\nu}}\right) \alpha^{2} \\
& -2\left(T_{\nu}+M_{R}^{*} Y_{\nu}\right) \alpha^{3}+3 Y_{\nu}^{2} \alpha^{4} .
\end{aligned}
$$

In order to avoid this direction in the potential from being equal or lower than zero, a sufficient way is requiring a negative discriminant in the solutions for $\alpha$ of the equation $V(\alpha)=0$. We get the following bound ${ }^{7}$

$\left(T_{\nu}+M_{R} Y_{\nu}\right)^{2} \leq 3\left(m_{H_{u}}^{2}+|\mu|^{2}+m_{\tilde{L}}^{2}+m_{\tilde{\nu}_{R}}^{2}+M_{R}^{2}+B_{\tilde{\nu}}\right) Y_{\nu}^{2}$.

\footnotetext{
${ }^{7}$ This result coincides with the one in Ref. [68] in the limit of vanishing $M_{R}$ and $B_{\nu}$ where the case of Dirac neutrinos has been studied. Note that their $A_{\nu}$ coincides with our $T_{\nu}$.
}

Writing $T_{\nu}=A_{\nu} Y_{\nu}$ we see immediately that

$$
\left(A_{\nu}+M_{R}\right)^{2} \leq 3\left(m_{H_{u}}^{2}+|\mu|^{2}+m_{\tilde{L}}^{2}+m_{\tilde{\nu}_{R}}^{2}+M_{R}^{2}+B_{\tilde{\nu}}\right)
$$

which implies it is $A_{\nu}$, and not $T_{\nu}$, who can be at most of the order of soft terms. Thus L-R mixing is not a safe procedure to solve the DM problem in this context. We note for completeness that this tree-level estimate can get significant loop corrections, as has been in shown in similar models $[69,70]$. However, the estimate gives the correct order of magnitude, which is sufficient in our case. We have explicitly checked that this estimate is correct within a factor two for a few points using the package VEVACIOUS [71].

\section{A $U(1)_{B-L}$ EXTENSION}

We have seen in the previous section that in the minimal model it is not possible to have a thermal $\tilde{\nu}_{R}$ as an LSP, as it will yield a too large contribution to $\Omega h^{2}$. We therefore seek an extension where the R-sneutrino can be a thermal relic, potentially giving the correct relic density, without impacting too much the high-energy and precision phenomenology, in particular in view of the collider constraints obtained in [26]. Several possibilities have been considered in a similar context in the literature: an additional $U(1)$ gauge factor [72-75], left-right symmetric models [76], the NMSSM $[77,78]$ or via additional F- and D-terms as they occur in models of hybrid inflation [79].

Taking a $U(1)_{B-L}$ extension as an example, we will show how this can be achieved in the parameter space we are interested in here. For this, we first briefly summarize the main aspects of the model presented in [80]. Here, the MSSM particle content is extended by three new types of superfields. First, one has a $B^{\prime}$ vector superfield associated to the $U(1)_{B-L}$ symmetry. Second, one adds two new Higgs-like chiral superfields, $\hat{\eta}$ and $\hat{\bar{\eta}}$, carrying $B-L$ number \pm 2 , whose scalar components break $U(1)_{B-L}$ and provide mass to the $Z^{\prime}$ boson. These chiral superfields will be called bileptons in the following. Note, that the symmetry breaking is such that $R$-parity is conserved. ${ }^{8}$ Finally, the anomaly-cancellation requires the existence of three right-handed neutrino superfields $\hat{\nu}_{R}$ [81]. These have masses around $10-100 \mathrm{GeV}$ if the $U(1)_{B-L}$ breaking scale is of the order $1-100 \mathrm{TeV}$.

The superpotential is given by

$$
\mathcal{W}=\mathcal{W}_{\mathrm{MSSM}}-\mu^{\prime} \hat{\eta} \hat{\bar{\eta}}+\left(Y_{x}\right)_{i j} \hat{\nu}_{R i} \hat{\nu}_{R j} \hat{\eta}+\left(Y_{\nu}\right)_{i j} \hat{L}_{i} \cdot \hat{H}_{u} \hat{\nu}_{R j}
$$

and one has the additional soft SUSY-breaking terms:

\footnotetext{
${ }^{8}$ This symmetry-breaking pattern is only a necessary but not a sufficient condition, as there could still be spontaneous $R$-parity violation due to a sneutrino vev [70].
} 
TABLE III. The values of the fixed parameters used in the numerical examples of BLSSM.

\begin{tabular}{lccccccc}
\hline \hline Parameter & Value & Parameter & Value & Parameter & Value & Parameter & Value \\
\hline$\mu^{\prime}$ & $500 \mathrm{GeV}$ & $M_{1}$ & $724 \mathrm{GeV}$ & $Y_{\nu, 11}$ & $10^{-8}$ & $Y_{x, 11}$ & $10^{-5}$ \\
$M_{Z}^{\prime}$ & $4.20 \mathrm{TeV}$ & $M_{2}$ & $1.19 \mathrm{TeV}$ & $\tan \beta$ & 20 & $Y_{x, 22}$ & $1.9 \times 10^{-2}$ \\
$m_{A^{0}}$ & $6.20 \mathrm{TeV}$ & $M_{3}$ & $3.10 \mathrm{TeV}$ & $g_{B-L}$ & 0.55 & $Y_{x, 33}$ & $1.9 \times 10^{-2}$ \\
\hline \hline
\end{tabular}

$$
\begin{aligned}
\mathcal{V}^{\text {soft }}= & \mathcal{V}_{\mathrm{MSSM}}^{\text {soft }}-M_{B B^{\prime}} \lambda_{\tilde{B}} \lambda_{\tilde{B}^{\prime}}-\frac{1}{2} M_{B^{\prime}} \lambda_{\tilde{B}^{\prime}} \lambda_{\tilde{B}^{\prime}}-m_{\eta}^{2}|\eta|^{2} \\
& -m_{\bar{\eta}}^{2}|\bar{\eta}|^{2}-B_{\mu^{\prime}} \eta \bar{\eta}-\left(m_{\tilde{\nu}_{R}}^{2}\right)_{i j} \tilde{\nu}_{R i}^{*} \tilde{R}_{R j} \\
& +\left(\left(T_{x}\right)_{i j} \tilde{\nu}_{R i} \tilde{\nu}_{R j} \eta+\left(T_{\nu}\right)_{i j} \tilde{L}_{i} \cdot H_{u} \tilde{\nu}_{R j}+\text { H.c. }\right) .
\end{aligned}
$$

Without loss of generality one can take $B_{\mu}$ and $B_{\mu^{\prime}}$ to be real, as in the MSSM. The extended gauge group breaks to $S U(3)_{C} \otimes U(1)_{e m}$ as beside the Higgs fields also the bileptons receive vevs, denoted by $v_{\eta}$ and $v_{\bar{\eta}}$, see Ref. [80] for details. We define $\tan \beta^{\prime}=\frac{v_{\eta}}{v_{\bar{\eta}}}$ in analogy to the ratio of the MSSM vevs. In the following we will neglect all effects concerning gauge kinetic mixing as they do not impact our findings, e.g., we will set $M_{B B^{\prime}}=0$.

As in the MSSM one can always get a SM-like light Higgs boson $h$, with mass of $125 \mathrm{GeV}$, assuming third generation squarks sufficiently heavy and having a sizable mixing in the stop sector. Beside $h$ this model contains a second scalar $h^{\prime}$, which is light provided $\tan \beta^{\prime}$ is close to 1 [80]. As the limits on $Z^{\prime}$ are in the multi-TeV range, this state is mainly an SM gauge-singlet, since the mixing scales like $v_{u} / \sqrt{v_{\bar{\eta}}^{2}+v_{\eta}^{2}}$. However, since $h$ can still have a non-negligible mixing with $h^{\prime}$, we use HiggsBounds [82] to check if its properties are compatible with the experimental results.

Neglecting mixing effects between the MSSM Higgs bosons and the bileptons, one gets for the mass of $h^{\prime}$ at tree level [80]:

$m_{h^{\prime}}^{2}=\frac{1}{2}\left(m_{Z^{\prime}}^{2}+m_{A^{0 \prime}}^{2}-\sqrt{\left(m_{Z^{\prime}}^{2}+m_{A^{0 \prime}}^{2}\right)^{2}-4 m_{Z^{\prime}}^{2} m_{A^{0}}^{2} \cos ^{2} 2 \beta^{\prime}}\right)$,

which has a structure similar to the MSSM case. ${ }^{9}$ From the above equation it follows that, for fixed $m_{Z^{\prime}}$ and $m_{A^{0 \prime}}$, one can control the value of $m_{h^{\prime}}$ by adjusting $\tan \beta^{\prime}$. In particular, $m_{h^{\prime}} \rightarrow 0$ when $\tan \beta^{\prime} \rightarrow 1$, and $m_{h^{\prime}} \rightarrow$ $\operatorname{Min}\left(m_{Z^{\prime}}, m_{A^{\prime \prime}}\right)$ for $\tan \beta^{\prime} \rightarrow 0$ or $\tan \beta^{\prime} \rightarrow \infty$.

Regarding the sneutrino sector, the $\Delta L=2$ operators, such as $\left(Y_{x}\right)_{i j} \hat{\nu}_{R i} \hat{\nu}_{R j} \hat{\eta}$, set, on the one hand, the mass scale of the right-handed neutrinos and lead, on the other hand, to a splitting of the complex sneutrino fields into their $C P$-even and $C P$-odd components, denoted $\tilde{\nu}^{S}$ and $\tilde{\nu}^{P}$, respectively. Neglecting left-right mixing and assuming $C P$ conservation, the masses of the R-sneutrinos can be expressed as [75]

\footnotetext{
${ }^{9} \mathrm{We}$ have checked that loop corrections are less important in this sector compared to the MSSM part.
}

$$
\begin{aligned}
m_{\tilde{\nu}^{S}}^{2} \simeq & m_{\tilde{\nu}_{R}}^{2}+m_{Z^{\prime}}^{2}\left(\frac{1}{4} \cos \left(2 \beta^{\prime}\right)+\frac{2 Y_{x}^{2}}{g_{B-L}^{2}} \sin ^{2} \beta^{\prime}\right) \\
& +m_{Z^{\prime}} \frac{\sqrt{2} Y_{x}}{g_{B-L}}\left(A_{x} \sin \beta^{\prime}-\mu^{\prime} \cos \beta^{\prime}\right), \\
m_{\tilde{\nu}^{P}}^{2} \simeq & m_{\tilde{\nu}_{R}}^{2}+m_{Z^{\prime}}^{2}\left(\frac{1}{4} \cos \left(2 \beta^{\prime}\right)+\frac{2 Y_{x}^{2}}{g_{B-L}^{2}} \sin ^{2} \beta^{\prime}\right) \\
& -m_{Z^{\prime}} \frac{\sqrt{2} Y_{x}}{g_{B-L}}\left(A_{x} \sin \beta^{\prime}-\mu^{\prime} \cos \beta^{\prime}\right) .
\end{aligned}
$$

Dark matter aspects of this model have been discussed in [75] where various candidates were evaluated, including sneutrinos. It has been shown that in case of a $C P$-even sneutrino it is possible to reproduce the correct relic density through a Higgs funnel mechanism, similar to other related models (see discussion in [18]). This mechanism is also applicable to $C P$-odd sneutrinos, being a consequence of the $D$-term and $F$-term couplings of the $h^{\prime}$ to the $\tilde{\nu}_{R}$. One issue that we want to address here is whether the Higgs funnel mechanism still explains the observed $\Omega h^{2}$ if we enforce a small or even negligible mass splitting between the scalar and pseudoscalar sneutrino states.

As it is well know, funnel regions imply particular relation between various masses, e.g., here we shall require $m_{\tilde{\nu}_{1}^{s}} \simeq m_{h^{\prime}} / 2$. Moreover, having a small splitting between

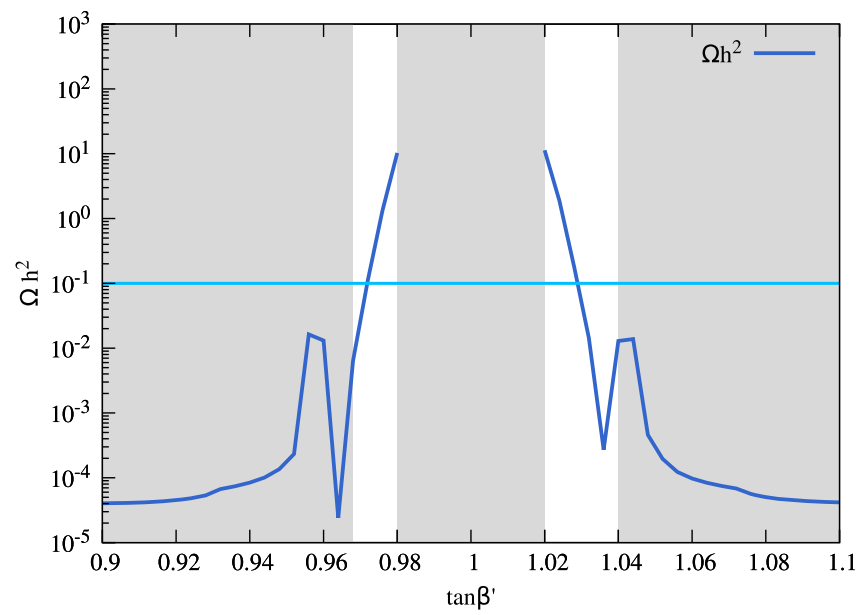

FIG. 5. Relic density $\Omega h^{2}$ due to sneutrino dark matter as a function of $\tan \beta^{\prime}$ for $m_{h^{\prime}}=2 m_{\tilde{\nu}_{1}^{s}}$ and $m_{\tilde{\nu}_{1}^{s}}-m_{\tilde{\nu}_{1}^{p}}=0.5 \mathrm{GeV}$. The other parameters are given in Table III. The two outer shaded areas are excluded by Higgs data whereas the middle one contains tachyonic states. 

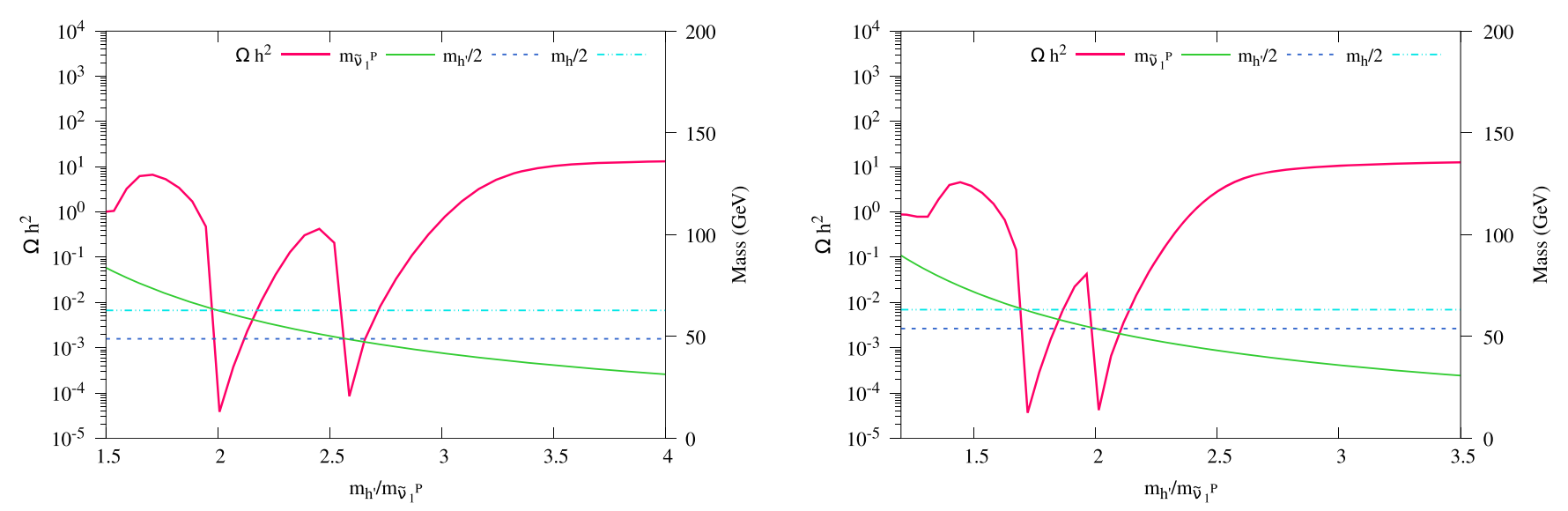

FIG. 6. Relic density $\Omega h^{2}$ due to sneutrino dark matter as a function of mass ratio $m_{h^{\prime}} / m_{\tilde{\nu}_{1}^{P}}$. We have set $m_{\tilde{\nu}^{s}}=m_{\tilde{\nu}_{1}^{p}}+0.5 \mathrm{GeV}$, $\tan \beta^{\prime}=0.972$ (left plot), and $\tan \beta^{\prime}=1.032$ (right plot). On the left (right) plot we find $m_{h^{\prime}}=98 \mathrm{GeV}\left(m_{h^{\prime}}=108 \mathrm{GeV}\right)$ and $m_{h} \simeq$ $125 \mathrm{GeV}$ in both cases. The dashed lines give half of the Higgs masses and the green one the sneutrino mass.

the scalar and pseudoscalar implies $A_{x} \sin \beta^{\prime} \simeq \mu^{\prime} \cos \beta^{\prime}$ as can be seen from Eqs. (3.4) and (3.5). We take as input the mass of the pseudoscalar sneutrino $m_{\tilde{\nu}_{1}^{p}}, \tan \beta^{\prime}, m_{Z}^{\prime}$ and fix the mass difference between $\tilde{\nu}_{1}^{S}$ and $\tilde{\nu}_{1}^{P}$ to $0.5 \mathrm{GeV}$. The values of the parameters, which we fix for these investigations, are given in Table III.

As an example we show $\Omega h^{2}$ as a function of $\tan \beta^{\prime}$ in Fig. 5. In the figure, at the boundaries of the evaluated values of $\tan \beta^{\prime}$, we find $m_{h^{\prime}} \approx 350 \mathrm{GeV}$ and a mixing of a few per-cent with the SM-like Higgs. Due to the mixing, several final states are allowed when the $\tilde{\nu}$ s annihilate through the funnel. For larger masses of $h^{\prime}$, the dominant annihilation is into $W^{ \pm}$pairs. As $\tan \beta^{\prime}$ approaches unity, the coannihilations are less efficient, as this channel gets suppressed and the $b \bar{b}$ final state becomes the most important one. Close to the central region, excluded due to tachyonic states, this channel is also closed and the dominant process is $\tilde{\nu} \tilde{\nu} \rightarrow \nu_{4} \nu_{4}$. Since it is suppressed by a small $Y_{x}$, the relic density increases considerably.

Thus, similar to the case of the MSSM, the Higgs funnel is only possible within a small strip in parameter space. In the case shown we find exactly two working values of $\tan \beta^{\prime}$. Interestingly enough, both are consistent with the experimental constraints, which we have checked using HiggsBounds. We note for completeness, that we find the soft parameter $m_{\tilde{\nu}_{R}}^{2}<0$ when $\tan \beta^{\prime}<1$. However, when matching this model to the one of the previous section, the D-term contribution from the $Z^{\prime}$ yields a positive value. ${ }^{10} \mathrm{In}$ the points explaining the relic density, $h^{\prime}$ has about one percent admixture with the SM-like Higgs boson.

The mixing among the Higgs bosons actually imply that the SM-like Higgs boson also yields a funnel region. This is

\footnotetext{
${ }^{10}$ We have checked that also in the region with $m_{\tilde{\nu}_{R}}^{2}<0$ the R-parity conserving minimum is the global one using VEVACIOUS [71].
}

shown in Fig. 6 where we vary the sneutrino mass parameters but keep the Higgs masses as at the points in Fig. 5 yielding the correct relic density. On the right (left) plot we display the case for $\tan \beta^{\prime}=0.972\left(\tan \beta^{\prime}=1.032\right)$. For these values, we actually have four (two) possible sneutrino masses for the smaller (larger) value of $\tan \beta^{\prime}$ which yield the correct relic density. These correspond to scenarios where either the gauge-singlet $h^{\prime}$ or the SM-like $h$ give rise to the Higgs funnel.

Last but not least we note for completeness that the $Z^{\prime}$ leads to a thermal distribution of the right-handed neutrinos in the early universe. However, in the preferred $\mathrm{keV}$ mass range for explaining the correct relic density, it is actually a warm dark matter candidate, which is excluded by observations of the Lyman- $\alpha$ forest [83-85]. To avoid this, as before, one needs to set a relatively large $M_{4}$, such that the $\nu_{4}$ decay quickly. Thus, the right-handed neutrino cannot contribute to the DM in this model.

\section{CONCLUSIONS}

In the last years there has been increasing interest in scenarios where a light right-handed neutrino could explain the observed relic dark matter density. This has also been motivated by the fact that no hint of a WIMP dark matter particle has been found so far. At the same time there has also been increasing interest in so-called natural SUSY scenarios where the higgsinos are the LSP. While in these scenarios one can avoid fine-tuning in the Higgs sector, one cannot explain the observed relic density as the Higgsinos annihilate very effectively.

We have thus combined both ideas and considered in a first step a supersymmetric scenario where the MSSM is extended by right-handed neutrino superfields where the Yukawa couplings are enhanced by an inverse seesaw structure. Here we attempted to address several questions: (i) Is it possible for SUSY loops to modify the lifetime of 
the lightest right-handed neutrino $\nu_{4}$ ? (ii) Is it possible for an R-sneutrino $\tilde{\nu}_{R}$ to be a thermal relic in such scenarios? (iii) If nonthermal, under which conditions would dark matter production occur?

We have found that the answer to the first two questions is negative in the more minimal model. (i) The LHC bounds on the SUSY particles are already so strong that the corresponding contributions to the decay $\nu_{4} \rightarrow \gamma \nu_{i}$ are suppressed, leading to a change of the partial widths of at most 10 percent with respect to the SM contribution. Consequently, since the $\nu_{4}$ should have a mass of a few $\mathrm{keV}$ to be an acceptable DM candidate, we find this scenario to be heavily constrained by cosmological data. (ii) In scenarios where $\tilde{\nu}_{R}$ are the LSP the Yukawa couplings can be at most of the order $10^{-5}$. This size is sufficient for the $\tilde{\nu}_{R}$ to thermalize, but the corresponding relic density is too large by many orders of magnitude. The only exception are regions where a sizable mixing with the $\tilde{\nu}_{L}$ is present, as has already been noticed in the literature. However, we have seen that these regions lead to charge breaking minima and, thus, are excluded.

We then find that the only possibility for R-sneutrino dark matter, in the minimal model, is (iii) having a nonthermal FIMP, produced via super-WIMP or freezein mechanisms. We find that the observed relic density can be reproduced through both mechanisms, with the superWIMP depending on the ratio of $\tilde{\nu}_{1}$ and NLSP masses. The freeze-in is in addition sensitive to $\left(Y_{\nu}\right)_{a 4}$. By modifying these parameters, one can make either super-WIMP or freeze-in dominant. We have also found that the NLSP lifetime can be significantly large, which could be in conflict with BBN.

Although the $\tilde{\nu}_{R}$ cannot be a thermal DM candidate in the $\operatorname{MSSM}+\tilde{\nu}_{R}$ framework, as discussed above, it might well be that this is only an effective model, e.g., an additional gauge group might be realized in the multi- $\mathrm{TeV}$ range. As an example we have considered the case of an additional $U(1)_{B-L}$ and extended previous studies by the mass hierarchy studied in the MSSM $+\tilde{\nu}_{R}$. The additional $Z^{\prime}$ in the multi-TeV range implies that the $\nu_{R}$ and $\tilde{\nu}_{R}$ get thermalized in the early Universe. In this class of models a second light Higgs boson is possible, which mixes somewhat with the SM-like Higgs boson. We have shown this allows for an explanation of the observed relic density via a Higgs funnel.

\section{ACKNOWLEDGMENTS}

We would like to thank Nicolas Bernal and Carlos Yaguna for discussions. T. F., Y. L., and W. P. have been supported by DFG, project no. PO-1337/7-1, and by DAAD, project no. 57395885. J. J. P. acknowledges funding by the Dirección de Gestión de la Investigación at Pontificia Universidad Católica del Perú (PUCP), through Grant No. DGI-2015-3-0026, and by the DAADCONCYTEC project no. 131-2017-FONDECYT.
[1] M. Papucci, J. T. Ruderman, and A. Weiler, Natural SUSY endures, J. High Energy Phys. 09 (2012) 035.

[2] D. Barducci, A. Belyaev, A. K. M. Bharucha, W. Porod, and V. Sanz, Uncovering natural supersymmetry via the interplay between the LHC and direct dark matter detection, J. High Energy Phys. 07 (2015) 066.

[3] E. Aprile et al. (XENON Collaboration), Dark Matter Search Results from a One Ton-Year Exposure of XENON1T, Phys. Rev. Lett. 121, 111302 (2018).

[4] M. Cirelli, N. Fornengo, and A. Strumia, Minimal dark matter, Nucl. Phys. B753, 178 (2006).

[5] P. Minkowski, $\mu \rightarrow e \gamma$ at a rate of one out of $10^{9}$ muon decays?, Phys. Lett. 67B, 421 (1977).

[6] T. Yanagida, Horizontal symmetry and masses of neutrinos, in The Unified Theory and the Baryon Number in the Universe Workshop, National Laboratory For High Energy Physics (KEK), 1979, edited by O. Sawada and A. Sugamoto (1979), pp. 95-99.

[7] R. N. Mohapatra and G. Senjanovic, Neutrino Mass and Spontaneous Parity Violation, Phys. Rev. Lett. 44, 912 (1980).

[8] M. Gell-Mann, P. Ramond, and R. Slansky, Complex spinors and unified theories, in Supergravity Workshop,
Stony Brook, New York, 1979, P. van Nieuwenhuizen and D. Z. Freedman (North-Holland, 1979), pp. 315-321.

[9] J. Schechter and J.W.F. Valle, Neutrino masses in $\mathrm{SU}(2) \times \mathrm{U}(1)$ theories, Phys. Rev. D 22, 2227 (1980).

[10] A. D. Medina, Higgsino-like dark matter from sneutrino late decays, Phys. Lett. B 770, 161 (2017).

[11] D. Hooper, J. March-Russell, and S. M. West, Asymmetric sneutrino dark matter and the $\Omega_{b} / \Omega_{D M}$ puzzle, Phys. Lett. B 605, 228 (2005).

[12] T. Asaka, K. Ishiwata, and T. Moroi, Right-handed sneutrino as cold dark matter, Phys. Rev. D 73, 051301(R) (2006).

[13] S. Gopalakrishna, A. de Gouvea, and W. Porod, Righthanded sneutrinos as nonthermal dark matter, J. Cosmol. Astropart. Phys. 05 (2006) 005.

[14] C. Arina and N. Fornengo, Sneutrino cold dark matter, a new analysis: Relic abundance and detection rates, J. High Energy Phys. 11 (2007) 029.

[15] V. Page, Non-thermal right-handed sneutrino dark matter and the $\Omega_{D M} / \Omega_{b}$ problem, J. High Energy Phys. 04 (2007) 021.

[16] G. Belanger, M. Kakizaki, E. K. Park, S. Kraml, and A. Pukhov, Light mixed sneutrinos as thermal dark matter, J. Cosmol. Astropart. Phys. 11 (2010) 017. 
[17] B. Dumont, G. Belanger, S. Fichet, S. Kraml, and T. Schwetz, Mixed sneutrino dark matter in light of the 2011 XENON and LHC results, J. Cosmol. Astropart. Phys. 09 (2012) 013.

[18] V. De Romeri and M. Hirsch, Sneutrino dark matter in lowscale seesaw scenarios, J. High Energy Phys. 12 (2012) 106.

[19] S. Banerjee, G. Bélanger, B. Mukhopadhyaya, and P. D. Serpico, Signatures of sneutrino dark matter in an extension of the CMSSM, J. High Energy Phys. 07 (2016) 095.

[20] K.-Y. Choi, J. Kim, and O. Seto, Thermal production of light Dirac right-handed sneutrino dark matter, Phys. Dark Universe 22, 96 (2018).

[21] A. Ghosh, T. Mondal, and B. Mukhopadhyaya, Right sneutrino with $\Delta L=2$ masses as nonthermal dark matter, Phys. Rev. D 99, 035018 (2019).

[22] A. Boyarsky, M. Drewes, T. Lasserre, S. Mertens, and O. Ruchayskiy, Sterile neutrino dark matter, Prog. Part. Nucl. Phys. 104, 1 (2019).

[23] A. de Gouvea, S. Gopalakrishna, and W. Porod, Stop decay into right-handed sneutrino LSP at hadron colliders, J. High Energy Phys. 11 (2006) 050.

[24] Z. Thomas, D. Tucker-Smith, and N. Weiner, Mixed sneutrinos, dark matter and the CERN LHC, Phys. Rev. D 77, 115015 (2008).

[25] D. Choudhury, S. K. Gupta, and B. Mukhopadhyaya, Right sneutrinos in a supergravity model and the signals of a stable stop at the Large Hadron Collider, Phys. Rev. D 78, 015023 (2008).

[26] N. Cerna-Velazco, T. Faber, J. Jones-Perez, and W. Porod, Constraining sleptons at the LHC in a supersymmetric lowscale seesaw scenario, Eur. Phys. J. C 77, 661 (2017).

[27] A. Chatterjee, J. Dutta, and S. K. Rai, Natural SUSY at LHC with right-sneutrino LSP, J. High Energy Phys. 06 (2018) 042 .

[28] S. Banerjee, G. Bélanger, A. Ghosh, and B. Mukhopadhyaya, Long-lived stau, sneutrino dark matter and right-slepton spectrum, J. High Energy Phys. 09 (2018) 143.

[29] J. A. Casas and A. Ibarra, Oscillating neutrinos and $\mu \rightarrow e \gamma$, Nucl. Phys. B618, 171 (2001).

[30] A. Donini, P. Hernandez, J. Lopez-Pavon, M. Maltoni, and T. Schwetz, The minimal $3+2$ neutrino model versus oscillation anomalies, J. High Energy Phys. 07 (2012) 161.

[31] A. Ibarra, E. Molinaro, and S. T. Petcov, TeV scale see-saw mechanisms of neutrino mass generation, the Majorana nature of the heavy singlet neutrinos and $(\beta \beta)_{0 \nu}$-decay, J. High Energy Phys. 09 (2010) 108.

[32] J. Lopez-Pavon, S. Pascoli, and C.-f. Wong, Can heavy neutrinos dominate neutrinoless double beta decay?, Phys. Rev. D 87, 093007 (2013).

[33] A. M. Gago, P. Hernández, J. Jones-Pérez, M. Losada, and A. M. Briceño, Probing the type I seesaw mechanism with displaced vertices at the LHC, Eur. Phys. J. C 75, 470 (2015).

[34] P. Hernández, J. Jones-Pérez, and O. Suárez-Navarro, Majorana vs Pseudo-Dirac neutrinos at the ILC, Eur. Phys. J. C 79, 220 (2019).

[35] P. F. de Salas, D. V. Forero, C. A. Ternes, M. Tortola, and J. W. F. Valle, Status of neutrino oscillations 2018: $3 \sigma$ hint for normal mass ordering and improved $C P$ sensitivity, Phys. Lett. B 782, 633 (2018).
[36] I. Esteban, M. C. Gonzalez-Garcia, A. HernandezCabezudo, M. Maltoni, and T. Schwetz, Global analysis of three-flavour neutrino oscillations: Synergies and tensions in the determination of $\theta_{2} 3, \delta_{C} P$, and the mass ordering, J. High Energy Phys. 01 (2019) 106.

[37] M. Tanabashi et al. (Particle Data Group), Review of particle physics, Phys. Rev. D 98, 030001 (2018).

[38] S. Dodelson and L. M. Widrow, Sterile-Neutrinos as Dark Matter, Phys. Rev. Lett. 72, 17 (1994).

[39] X.-D. Shi and G. M. Fuller, A New Dark Matter Candidate: Nonthermal Sterile Neutrinos, Phys. Rev. Lett. 82, 2832 (1999).

[40] K. C. Y. Ng, B. M. Roach, K. Perez, J. F. Beacom, S. Horiuchi, R. Krivonos, and D. R. Wik, New constraints on sterile neutrino dark matter from NuSTAR M31 observations, Phys. Rev. D 99, 083005 (2019).

[41] M. Drewes, On the minimal mixing of heavy neutrinos, arXiv: 1904.11959.

[42] H. E. Haber and D. Wyler, Radiative neutralino decay, Nucl. Phys. B323, 267 (1989).

[43] A. C. Vincent, E. F. Martinez, P. Hernández, M. Lattanzi, and O. Mena, Revisiting cosmological bounds on sterile neutrinos, J. Cosmol. Astropart. Phys. 04 (2015) 006.

[44] B. Shakya and J. D. Wells, Sterile neutrino dark matter with supersymmetry, Phys. Rev. D 96, 031702 (2017).

[45] G. Bertone, D. Hooper, and J. Silk, Particle dark matter: Evidence, candidates and constraints, Phys. Rep. 405, 279 (2005).

[46] J. L. Feng, A. Rajaraman, and F. Takayama, Superweakly Interacting Massive Particles, Phys. Rev. Lett. 91, 011302 (2003).

[47] J. L. Feng, A. Rajaraman, and F. Takayama, SuperWIMP dark matter signals from the early universe, Phys. Rev. D 68, 063504 (2003).

[48] T. Asaka, K. Ishiwata, and T. Moroi, Right-handed sneutrino as cold dark matter of the universe, Phys. Rev. D 75, 065001 (2007).

[49] L. J. Hall, K. Jedamzik, J. March-Russell, and S. M. West, Freeze-in production of FIMP dark matter, J. High Energy Phys. 03 (2010) 080.

[50] G. Bélanger et al., LHC-friendly minimal freeze-in models, J. High Energy Phys. 02 (2019) 186.

[51] F. Staub, SARAH, arXiv:0806.0538.

[52] F. Staub, SARAH 4: A tool for (not only SUSY) model builders, Comput. Phys. Commun. 185, 1773 (2014).

[53] F. Staub, SARAH 3.2: Dirac Gauginos, UFO output, and more, Comput. Phys. Commun. 184, 1792 (2013).

[54] F. Staub, Automatic calculation of supersymmetric renormalization group equations and self energies, Comput. Phys. Commun. 182, 808 (2011).

[55] F. Staub, From superpotential to model files for FeynArts and CalcHep/CompHep, Comput. Phys. Commun. 181, 1077 (2010).

[56] W. Porod, SPheno, A program for calculating supersymmetric spectra, SUSY particle decays and SUSY particle production at e+ e- colliders, Comput. Phys. Commun. 153, 275 (2003).

[57] W. Porod and F. Staub, SPheno 3.1: Extensions including flavour, $C P$-phases and models beyond the MSSM, Comput. Phys. Commun. 183, 2458 (2012). 
[58] F. Staub, T. Ohl, W. Porod, and C. Speckner, A tool box for implementing supersymmetric models, Comput. Phys. Commun. 183, 2165 (2012).

[59] G. Bélanger, F. Boudjema, A. Goudelis, A. Pukhov, and B. Zaldivar, micrOMEGAs5.0: Freeze-in, Comput. Phys. Commun. 231, 173 (2018).

[60] M. Garny and J. Heisig, Interplay of super-WIMP and freezein production of dark matter, Phys. Rev. D 98, 095031 (2018).

[61] T. Asaka, M. Shaposhnikov, and A. Kusenko, Opening a new window for warm dark matter, Phys. Lett. B 638, 401 (2006).

[62] M. Kawasaki, K. Kohri, and T. Moroi, Big-bang nucleosynthesis and hadronic decay of long-lived massive particles, Phys. Rev. D 71, 083502 (2005).

[63] K. Ishiwata, M. Kawasaki, K. Kohri, and T. Moroi, Righthanded sneutrino dark matter and big-bang nucleosynthesis, Phys. Lett. B 689, 163 (2010).

[64] M. Kawasaki, K. Kohri, T. Moroi, and Y. Takaesu, Revisiting big-bang nucleosynthesis constraints on long-lived decaying particles, Phys. Rev. D 97, 023502 (2018).

[65] N. Aghanim et al. (Planck Collaboration), Planck 2018 results. VI. Cosmological parameters, arXiv:1807.06209.

[66] N. Arkani-Hamed, L. J. Hall, H. Murayama, D. Smith, and N. Weiner, Small neutrino masses from supersymmetry breaking, Phys. Rev. D 64, 115011 (2001).

[67] J. A. Casas, A. Lleyda, and C. Munoz, Strong constraints on the parameter space of the MSSM from charge and color breaking minima, Nucl. Phys. B471, 3 (1996).

[68] M. Kakizaki, E.-K. Park, J.-h. Park, and A. Santa, Phenomenological constraints on light mixed sneutrino dark matter scenarios, Phys. Lett. B 749, 44 (2015).

[69] J. E. Camargo-Molina, B. O'Leary, W. Porod, and F. Staub, Stability of the CMSSM against sfermion VEVs, J. High Energy Phys. 12 (2013) 103.

[70] J. E. Camargo-Molina, B. O'Leary, W. Porod, and F. Staub, The stability of R-parity in supersymmetric models extended By $U(1)_{B-L}$, Phys. Rev. D 88, 015033 (2013).

[71] J. E. Camargo-Molina, B. O'Leary, W. Porod, and F. Staub, Vevacious: A tool for finding the global minima of one-loop effective potentials with many scalars, Eur. Phys. J. C 73, 2588 (2013).

[72] H.-S. Lee, K. T. Matchev, and S. Nasri, Revival of the thermal sneutrino dark matter, Phys. Rev. D 76, 041302(R) (2007).
[73] G. Belanger, J. Da Silva, and A. Pukhov, The right-handed sneutrino as thermal dark matter in U(1) extensions of the MSSM, J. Cosmol. Astropart. Phys. 12 (2011) 014.

[74] P. Bandyopadhyay, E. J. Chun, and J.-C. Park, Right-handed sneutrino dark matter in $\mathbf{U}(\mathbf{1})^{\prime}$ seesaw models and its signatures at the LHC, J. High Energy Phys. 06 (2011) 129.

[75] L. Basso, B. O'Leary, W. Porod, and F. Staub, Dark matter scenarios in the minimal SUSY B-L model, J. High Energy Phys. 09 (2012) 054.

[76] M. Frank, B. Fuks, K. Huitu, S. K. Rai, and H. Waltari, Resonant slepton production and right sneutrino dark matter in left-right supersymmetry, J. High Energy Phys. 05 (2017) 015.

[77] D. G. Cerdeno and O. Seto, Right-handed sneutrino dark matter in the NMSSM, J. Cosmol. Astropart. Phys. 08 (2009) 032.

[78] D. G. Cerdeno, J.-H. Huh, M. Peiro, and O. Seto, Very light right-handed sneutrino dark matter in the NMSSM, J. Cosmol. Astropart. Phys. 11 (2011) 027.

[79] F. Deppisch and A. Pilaftsis, Thermal right-handed sneutrino dark matter in the $\mathrm{F}(\mathrm{D})$-term model of hybrid inflation, J. High Energy Phys. 10 (2008) 080.

[80] B. O'Leary, W. Porod, and F. Staub, Mass spectrum of the minimal SUSY B-L model, J. High Energy Phys. 05 (2012) 042.

[81] V. Barger, P. Fileviez Perez, and S. Spinner, Minimal Gauged $U(1)_{(B-L)}$ Model with Spontaneous R-Parity Violation, Phys. Rev. Lett. 102, 181802 (2009).

[82] P. Bechtle, O. Brein, S. Heinemeyer, O. Stål, T. Stefaniak, G. Weiglein, and K. E. Williams, HiggsBounds-4: Improved tests of extended Higgs sectors against exclusion bounds from LEP, the Tevatron and the LHC, Eur. Phys. J. C 74, 2693 (2014).

[83] M. Viel, G. D. Becker, J. S. Bolton, and M. G. Haehnelt, Warm dark matter as a solution to the small scale crisis: New constraints from high redshift Lyman- $\alpha$ forest data, Phys. Rev. D 88, 043502 (2013).

[84] V. Iršič et al., New constraints on the free-streaming of warm dark matter from intermediate and small scale Lyman- $\alpha$ forest data, Phys. Rev. D 96, 023522 (2017).

[85] J. Baur, N. Palanque-Delabrouille, C. Yeche, A. Boyarsky, O. Ruchayskiy, É. Armengaud, and J. Lesgourgues, Constraints from Ly- $\alpha$ forests on non-thermal dark matter including resonantly-produced sterile neutrinos, J. Cosmol. Astropart. Phys. 12 (2017) 013. 